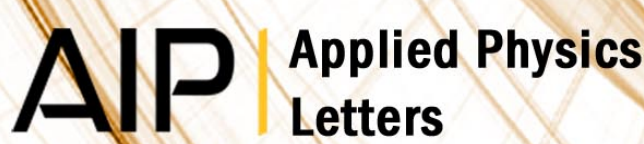

\section{A monolithic optical sensor based on whispering-gallery modes in polystyrene microspheres}

Julie Lutti, Wolfgang Langbein, and Paola Borri

Citation: Appl. Phys. Lett. 93, 151103 (2008); doi: 10.1063/1.2998652

View online: http://dx.doi.org/10.1063/1.2998652

View Table of Contents: http://apl.aip.org/resource/1/APPLAB/v93/i15

Published by the American Institute of Physics.

\section{Related Articles}

Bloch surface waves-controlled fluorescence emission: Coupling into nanometer-sized polymeric waveguides APL: Org. Electron. Photonics 5, 39 (2012)

Bloch surface waves-controlled fluorescence emission: Coupling into nanometer-sized polymeric waveguides Appl. Phys. Lett. 100, 063305 (2012)

Identifying and evaluating organic nonlinear optical materials via molecular moments

J. Appl. Phys. 111, 033512 (2012)

Elastomeric silicone substrates for terahertz fishnet metamaterials

Appl. Phys. Lett. 100, 061101 (2012)

Functionalizing the rear scatterer in a luminescent solar concentrator

J. Renewable Sustainable Energy 4, 013103 (2012)

\section{Additional information on Appl. Phys. Lett.}

Journal Homepage: http://apl.aip.org/

Journal Information: http://apl.aip.org/about/about_the_journal

Top downloads: http://apl.aip.org/features/most_downloaded

Information for Authors: http://apl.aip.org/authors

\section{ADVERTISEMENT}

\section{ACCELERATE AMBER AND NAMD BY $5 X$.}

NVIDIA TRYIT ONA FREE, REMOTELYYHOSTED CLUSTER. 


\title{
A monolithic optical sensor based on whispering-gallery modes in polystyrene microspheres
}

\author{
Julie Lutti, Wolfgang Langbein, and Paola Borri ${ }^{\text {a) }}$ \\ School of Physics and Astronomy and School of Biosciences, Cardiff University, The Parade, Cardiff CF24 \\ 3AA, United Kingdom
}

(Received 1 September 2008; accepted 20 September 2008; published online 13 October 2008)

\begin{abstract}
We experimentally demonstrate a monolithic sensor based on sharp whispering-gallery optical resonances of polystyrene microspheres in water attached onto an optical coupler via a solid separation layer index matched to water. A $Q$-factor of $0.9 \times 10^{6}$ is measured on a microsphere of $44 \mu \mathrm{m}$ diameter with a $650 \mathrm{~nm}$ thick separation layer. As a proof of principle of the sensing capabilities, we measure the response to refractive index changes and infer a shot-noise limited precision of $1 \times 10^{-8}$ refractive index units. (c) 2008 American Institute of Physics.
\end{abstract}

[DOI: $10.1063 / 1.2998652]$

Optical microresonators have been recently proposed as a powerful method to achieve label-free detection of target material for chemical and biosensing applications. In particular, whispering-gallery-mode (WGM) sensors exploit sharp photonic resonances arising from light circumnavigating repeatedly near the surface of a dielectric microsphere (or microcylinder) by quasitotal internal reflection. Small changes of the refractive index in the region surrounding the microresonator, for example due to the attachment of target biomolecules at its surface, can be probed with high sensitivity via the corresponding frequency shift in the WGM resonances. ${ }^{1,2}$

A key issue of a WGM sensor is the coupling of light in and out of the resonator, which can be realized efficiently by locating an optical element (e.g., a fiber or prism) in its vicinity within the evanescent field of the WGM. ${ }^{3}$ The control of the distance between such optical coupler and the resonator is crucial to maintain high cavity $Q$-factors (which produce high sensor sensitivity) eventually limited only by the intrinsic scattering losses inside the resonators rather than by photon escape into the coupler. ${ }^{3}$ In practice this can be achieved using mechanical positioning tools via, e.g., piezoelectric stages with nanometer accuracy. However for the possible commercial exploitation of such senors a monolithic device integrating the resonator and the optical coupler at an optimum distance in a way that can be easily repeated and scaled for mass production is highly desirable.

In a previous communication, we reported the measurement of WGM resonances in polystyrene microspheres (PMs) held in water by optical tweezers. By controlling the distance of the resonator to a high-index glass half-ball coupler via the optical tweezers, we demonstrated a $Q$-factor of $4 \times 10^{6}$, promising for sensing applications. ${ }^{4}$ In this work, we have realized a monolithic device exploiting WGMs from PMs attached to a high-index glass coupler by a solid separation layer of controlled thickness and index matched to water, which allowed us to preserve the high $Q$-factor. With this device we demonstrate sensing to refractive index changes, and infer a shot-noise sensitivity limit of $10^{-8}$.

The sample structure is sketched in Fig. 1 and consists of a high-index (1.826) glass coupler onto which Cytop ${ }^{\circledR}$ (Asahi Glass), a perfluoropolymer chosen for its refractive

${ }^{a)}$ Electronic mail: borrip@cardiff.ac.uk. index (1.337) close to that of water (1.328), is used to form a solid separation layer as well as to attach the PMs via a two-step temperature curing procedure. ${ }^{5}$ We used several optical techniques to characterize the penetration of the PMs into the Cytop layer and the distance to the optical coupler. Imaging the samples in oil of index 1.52 by optical transmission microscopy [Fig. 2(a)] allowed us to see the attachment region, from which we deduce $(4.3 \pm 0.2) \%$ coverage of the PM circumference by Cytop. By imaging the sample in water instead, which is nearly index matched to Cytop, we observed no sign of defects or bead deformation in the attachment region [Fig. 2(b)]. From reflectometry spectra [Fig. 2(e)], the Cytop layer was measured to have a thickness of $770 \mathrm{~nm}$. Finally, we used differential interference contrast (DIC) microscopy [Fig. 2(d)] of the attachment region after removal of the bead to get quantitative information about the depth and the shape of the print. We infer that the separation between the PMs and the glass coupler is $650 \pm 20 \mathrm{~nm}$. A separation of this magnitude is needed to observe sharp WGMs. In fact in our previous work ${ }^{4}$ we showed that the WGM broadening due to photon escape into the coupler optical modes is exponentially decreasing with increasing distance with the decay length of the evanescent field intensity of the WGM $(\sim 90 \mathrm{~nm})$.

In order to optically excite WGM resonances by frustrated total internal reflection at the glass/Cytop interface

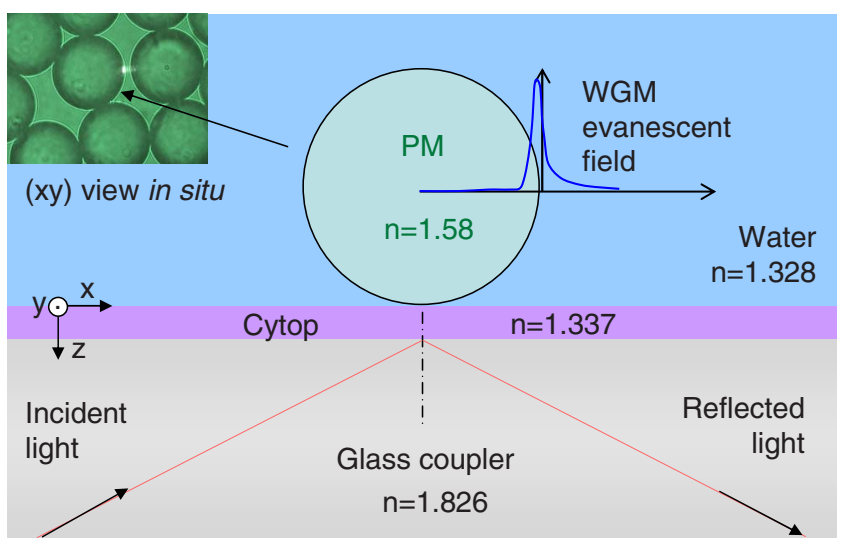

FIG. 1. (Color online) Sketch (not in scale) of the monolithic optical sensor with indicated materials and refractive indices. PM: Polystyrene microsphere. The inset shows a transmission image of the sample inside the flow chamber. Some scattering of the WGM light from the resonator is visible. 

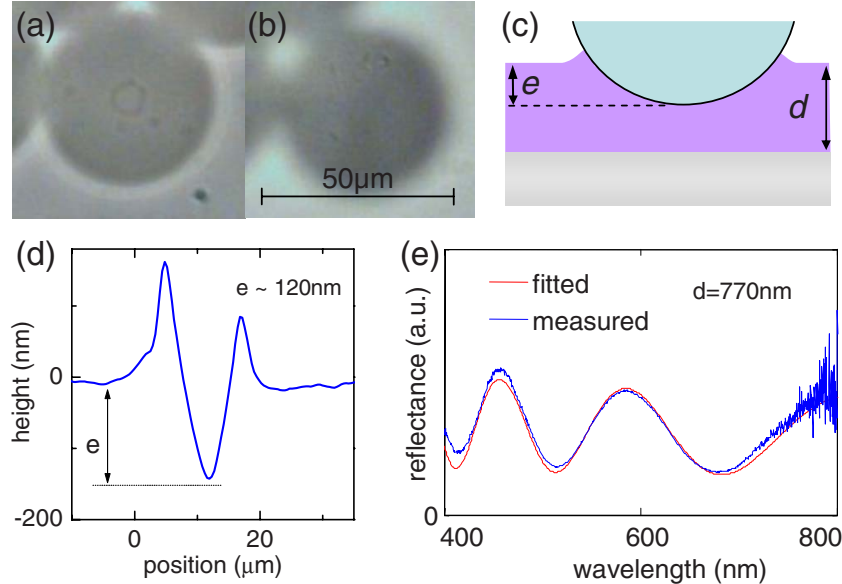

FIG. 2. (Color online) Transmission microscopy images with focus at the Cytop surface of a PM sample immersed in (a) oil or (b) water, (c) sketch of PM attachment region, (d) Cytop height profile in the attachment region, measured by DIC with the PM removed, (e) reflectance spectrum of the sample outside the attachment region in air.

(see Fig. 1) the sample was mounted on a high-index cut ball using an index-matching medium so that a half-ball coupler of $n=1.826$ index with the PM in the center is obtained. ${ }^{4}$ Optical excitation is provided by a wavelength-scanned distributed feedback (DFB) laser with a linewidth of $16 \mathrm{neV}$. Transverse electric (TE) and transverse magnetic (TM) WGM resonances couple, respectively, to the excitation polarized along $y$ and in the $x z$ plane (see axis system in Fig. $1)$. Let us denote by $E_{\mathrm{TE}}$ and $E_{\mathrm{TM}}$ the related field components in the reflected beam, which is divided by a Wollaston prism into two orthogonal polarization components. Their intensity difference is then measured using two photodiodes. Two detection schemes are used to measure resonances in $E_{\mathrm{TE}}$ while exploiting $E_{\mathrm{TM}}$ as a reference. In the "intensity" detection, we choose the detected components along $E_{\mathrm{TE}}$ and $E_{\mathrm{TM}}$ and evaluate their intensity difference normalized to the TM intensity,

$$
S_{I}=\frac{\left|E_{\mathrm{TE}}\right|^{2}-\left|E_{\mathrm{TM}}\right|^{2}}{\left|E_{\mathrm{TM}}\right|^{2}} .
$$

In the "interference" detection, we choose the detected polarizations at $45^{\circ}$ to $E_{\mathrm{TE}}$ and $E_{\mathrm{TM}}$ and thus measure the interference between the two fields,

$$
S_{E}=\frac{\left|E_{\mathrm{TM}}-E_{\mathrm{TE}}\right|^{2}-\left|E_{\mathrm{TM}}+E_{\mathrm{TE}}\right|^{2}}{2\left|E_{\mathrm{TM}}\right|^{2}} .
$$

In both cases, the excitation is adjusted so that the signal is zero out of resonance (balanced detection). For a TE resonance of frequency $\omega_{0}$ and polarization decay rate $\gamma$ the amplitude of the field in the reflected wave can be written as

$$
E_{\mathrm{TE}}=R E_{0}(\omega)\left(1+\frac{A \gamma}{i\left(\omega-\omega_{0}\right)-\gamma}\right) e^{i \psi},
$$

where $R$ is the reflection coefficient out of resonance, $E_{0}$ is the amplitude of the incident wave, $A$ is a real positive coefficient, and $\psi$ is a relative phase that can be adjusted by polarization optics in the excitation. The signal measured in the intensity detection [Eq. (1)] is then given by [using $\left.E_{\mathrm{TM}}=R E_{0}(\omega)\right]$,

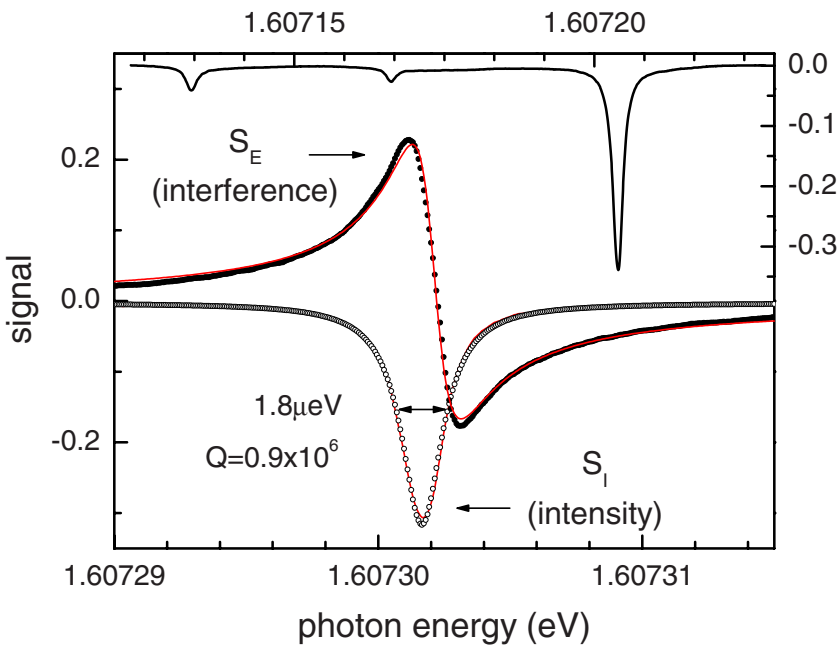

FIG. 3. (Color online) $\mathrm{TE} 1_{271}$ WGM resonance of the PM in water attached to the glass coupler, measured with the intensity (open circles) and the interference (closed circles) detections, and fitted lineshapes (solid lines). The detected subensemble of $\mathrm{TE} 1_{271}$ azimuthal modes is shown on the top axis in intensity detection.

$$
S_{I}=\frac{A(A-2) \gamma^{2}}{\gamma^{2}+\left(\omega-\omega_{0}\right)^{2}} .
$$

In the interference detection, the polarization optics is adjusted to $\psi=\pi / 2$ so that from Eq. (2) we find,

$$
S_{E}=\frac{-2 A \gamma\left(\omega-\omega_{0}\right)}{\gamma^{2}+\left(\omega-\omega_{0}\right)^{2}} .
$$

Figure 3 shows a TE resonance measured with both detection schemes of a PM attached to Cytop and immersed in water. As indicated by the notation $\mathrm{TE} 1_{271}$, we have classified the WGM as mode order $n=1$, and determined the mode number $\ell=271$ and the bead diameter $(43.8 \mu \mathrm{m})$ by comparing several WGM resonance energies with Mie theory. ${ }^{6}$ Both spectra are well described by Eqs. (4) and (5) with the same fitting parameters $A=0.2$ and $2 \gamma=1.8 \mu \mathrm{eV}$, plus a small offset to correct for nonperfect balancing of the detection. The dip in the intensity spectrum, due to destructive interference with the light that returns from the resonator, has an amplitude of 0.3 . Accordingly, at resonance $30 \%$ of the optical power spectrum is transferred into other modes mainly due to scattering by the microresonator. We emphasize that in the interference case one could detect the phase change induced by the WGM even when no amplitude change would occur such as in the regime of "overcoupling" where most of the exciting light couples back into the reflected mode. ${ }^{3}$ Moreover since the dispersive lineshape exhibits a steep slope at resonance, by differentiating one obtains a twofold sharper resonance linewidth, which can be exploited for increased sensitivity in the detection of WGM energy shifts.

PMs from the same batch, held in water by optical tweezers at a far enough distance from the glass surface so that the linewidth was not influenced by the coupler, showed WGM linewidths for $n=1$ resonances in a range from 0.4 to $1.5 \mu \mathrm{eV}$ (corresponding to $Q$-factors from $4 \times 10^{6}$ to 1.1 $\left.\times 10^{6}\right)$. The measured WGM linewidth of the microsphere attached to the coupler of $1.8 \mu \mathrm{eV}\left(Q=0.9 \times 10^{6}\right)$ with large $(30 \%)$ visibility hence demonstrates that the attachment procedure has not significantly deteriorated the cavity $Q$-factor. When the PMs were free to rotate in the optical trap, ${ }^{4}$ the 


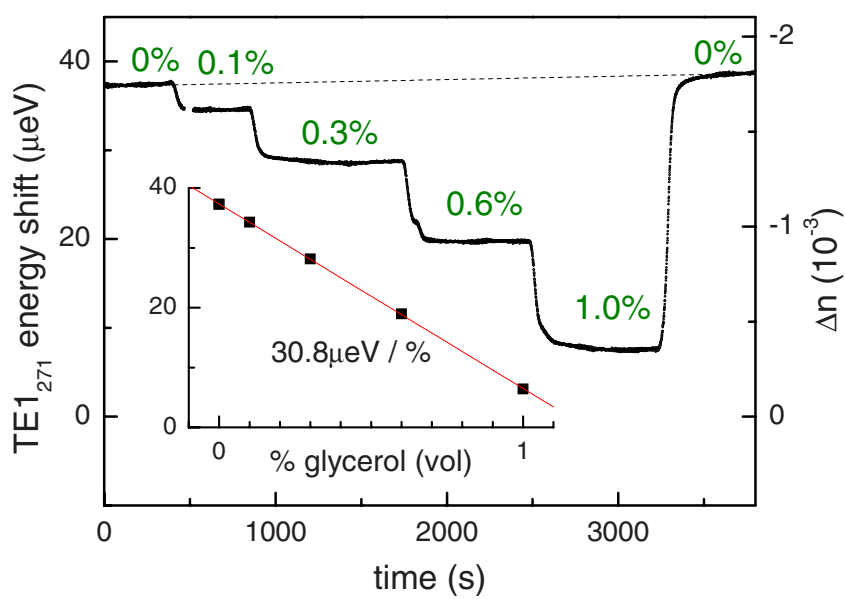

FIG. 4. (Color online) Refractive index sensogram: TE $1_{271}$ WGM energy shift upon change in the refractive index of the surrounding medium by a water/glycerol mixture at the indicated glycerol concentration. The inset shows the fitted responsivity. The right axis indicates the corresponding refractive index change in the medium.

spatially localized excitation would couple to different subensembles of azimuthal modes, arising from the nonperfect sphericity of the resonator, which lifts the $(2 \ell+1)$-fold degeneracy so that the weight of different modes in the detected spectrum is changing with time. On the PMs attached to the coupler we also observe a few azimuthal modes (see top of Fig. 3). However since the PMs are fixed, the weight of these resonances is stable over time. Theoretical predictions indicate that the separation layer, which is slightly indexed mismatched (0.009) relative to water, introduces an energy splitting of the same order of magnitude as observed in our experiment.

As a proof of principle of the sensing capabilities, the response of the WGM resonances to a change in the refractive index in the surrounding medium was tested in the intensity detection mode. Different mixtures of glycerol in water at volume concentrations ranging from $0 \%$ to $1 \%$ were injected in the flow chamber where the sample is placed. The chamber was temperature stabilized at $21^{\circ} \mathrm{C}$ by a chiller. The sensor responsivity to temperature changes was measured to be $(7 \pm 1) \mu \mathrm{eV} / \mathrm{K}$. Figure 4 shows the resonance energy of the TE $1_{271}$ WGM (obtained from the Lorentzian fit as in Fig. 3) shifting with time as the medium is modified. The sensogram shows clear steps corresponding to refractive index increases as the glycerol concentration is increased. Additionally, a slow drift toward higher energy of $3.810^{-4} \mu \mathrm{eV} / \mathrm{s}$ is indicated by the dashed line. The origin of this drift is so far unclear and is possibly due to desorption of surfactants from the PM. After correction by this linear drift, the energies for the plateaux are plotted in the inset of Fig. 4, showing a linear relationship between energy shift and glycerol concentration with a slope of $30.8 \mu \mathrm{eV} / \%$. With a refractive index for glycerol of $1.44 \times 10^{-3} / \mathrm{vol} \%{ }^{7}$ we deduce a responsivity of the sensor of $(-21.4 \pm 0.8) \mathrm{meV} / \mathrm{RIU}$, the error reflecting pipetting, and inital chemical composition inaccuracies. This is comparable to the theoretical value of $(-21.3 \pm 1) \mathrm{meV} / \mathrm{RIU}$ predicted by Mie theory ${ }^{6}$ for a PM of $1.58 \pm 0.05$ refractive index and $(43.8 \pm 0.02) \mu \mathrm{m}$ diameter, taking into account a $4.3 \%$ coverage of the PM by Cytop over the extent of the optical mode.

In our experiment, the precision of the measured resonance energy is mainly limited by source wavelength fluc- tuations (spectral jitter) of about $30 \mathrm{neV}$, caused by fluctuations of the DFB laser diode temperature and current, which effectively limits the sensitivity to $1.5 \times 10^{-6}$ RIU. The uncertainty due to intensity noise in the data is only $1 \mathrm{neV}$, which corresponds to $5 \times 10^{-8} \mathrm{RIU}$ as minimum detectable refractive index change.

For biosensing applications, the microsphere should be functionalized to interact with a specific analyte (via, e.g., antibody-antigen interaction). To isolate the effect of specific binding, the measurement of the WGM energy should be referenced to that of an unfunctionalized PM. Such referencing could also be effective in reducing the uncertainty caused by spectral jitter. To investigate this point, we have measured the energy difference between two adjacent modes on a similarly prepared sample. The resonances were two azimuthal modes $6 \mu \mathrm{eV}$ apart, of $1.8 \mu \mathrm{eV}$ linewidths and depths of about 0.1 . We measured the energy difference with a standard deviation of $7 \mathrm{neV}$ ( $5 \mathrm{neV}$ in the individual resonance), corresponding to a sensitivity of $3 \times 10^{-7}$ RIU, improved compared to the nonreferenced case. If the spectral jitter would be completely overcome (by, e.g., referencing two WGMs having identical resonant energies, thus experiencing exactly the same common mode noise), we estimate a shotnoise sensitivity limit for the same experimental conditions as in the measurements in Fig. 4 of $1 \times 10^{-8}$ RIU, even better than the measured intensity-noise limit, which was affected by the resolution in the digitization of the data. For biosensing applications, using the expression developed in Ref. 8, we infer a protein detection sensitivity of $5 \times 10^{-3} \mathrm{pg} / \mathrm{mm}^{2}$ in terms of surface mass loading and $0.03 \mathrm{fg}$ in terms of minimum detectable total mass loading. This sensitivity surpasses significantly the best specified in commercially available surface-plasmon-resonance methods.

In conclusion we have demonstrated an optical sensor device based on the detection of sharp whispering-gallery modes in PMs attached to a glass coupler via a solid separation layer index matched to water. The development of such monolithic device still exhibiting the high quality factor needed for highly sensitive detection, without the need to use piezoelectric positioning mechanisms to achieve controlled distance of the microresonator to the optical coupler, is a key step toward commercial exploitation and mass production of integrated WGM sensor devices.

This work is funded by the UK Ministry of Defense (Dstl) under Contract No. DSTLX-1000002440.

${ }^{1}$ F. Vollmer, D. Braun, A. Libchaber, M. Khoshsima, I. Teraoka, and S. Arnold, Appl. Phys. Lett. 80, 4057 (2002).

${ }^{2}$ A. M. Armani, R. P. Kulkarni, S. E. Fraser, R. C. Flagan, and K. J. Vahala, Science 317, 783 (2007).

${ }^{3}$ M. L. Gorodetsky and V. S. Ilchenko, J. Opt. Soc. Am. B 16, 147 (1999). ${ }^{4}$ J. Lutti, W. Langbein, and P. Borri, Appl. Phys. Lett. 91, 141116 (2007). ${ }^{5} \mathrm{PMs}$ (Polysciences, Inc.) are from a batch of average diameter $43.3 \mu \mathrm{m}$ and standard deviation $2.3 \mu \mathrm{m}$. A high-index (1.826) glass plate of 0.5 $\mathrm{mm}$ thickness is first spin coated at $3300 \mathrm{rpm}$ for $40 \mathrm{~s}$, with a layer of Cytop ${ }^{\mathrm{TM}}$ at $7 \%$ polymer concentration, and then cured at $180^{\circ} \mathrm{C}$ for $1 \mathrm{~h}$, giving a polymer thickness of about $550 \mathrm{~nm}$. After curing, another layer of Cytop solution at $5 \%$ polymer concentration is spin coated at $4200 \mathrm{rpm}$, and a drop of water containing the PMs is added on top. The sample is then reheated at $115^{\circ} \mathrm{C}$ for $10 \mathrm{~min}$.

${ }^{6}$ C. C. Lam, P. T. Leung, and K. Young, J. Opt. Soc. Am. B 9, 1585 (1992). ${ }^{7}$ Calibration table from Mettler Toledo.

${ }^{8}$ S. Arnold, M. Khoshsima, I. Teraoka, S. Holler, and F. Vollmer, Opt. Lett. 28, 272 (2003). 